\title{
La passion comme source identitaire et moteur d'engagement environnemental
}

Anne-Sophie Gousse-Lessard, Daniel Lalande et Robert J. Vallerand

\author{
(2) OpenEdition \\ Journals \\ Édition électronique \\ URL : http://journals.openedition.org/ere/549 \\ DOI : $10.4000 /$ ere.549 \\ ISSN : 2561-2271 \\ Éditeur \\ Centr'ERE
}

Référence électronique

Anne-Sophie Gousse-Lessard, Daniel Lalande et Robert J. Vallerand, « La passion comme source identitaire et moteur d'engagement environnemental », Éducation relative à l'environnement [En ligne], Volume 12 | 2015, mis en ligne le 20 mai 2015, consulté le 10 décembre 2020. URL : http:// journals.openedition.org/ere/549; DOI : https://doi.org/10.4000/ere.549 


\title{
La passion comme source identitaire et moteur d'engagement environnemental
}

\author{
Anne-Sophie Gousse-Lessard, Daniel Lalande et Robert J. Vallerand
}

1 Les connaissances sur l'environnement se multiplient. Divers savoirs comme les savoirfaire et savoir-être, se transmettent, se partagent. Les populations sont de plus en plus sensibilisées aux problématiques environnementales qui touchent leurs communautés. Pourtant, tous ne s'impliquent pas de la même manière et avec la même intensité en matière d'environnement. Pourquoi certaines personnes choisissent-elles de s'engager pleinement, concrètement et de façon (pro)active afin de contribuer à l'écodéveloppement des sociétés? Quels sont les déterminants de leur motivation? Comment peut-on encourager ce type d'implication? La passion pourrait bien faire partie de la réponse. Appuyée par les résultats d'une étude préliminaire issue de la psychologie sociale et environnementale, une réflexion sera entamée sur les liens entre identité écologique, passion et conduite écocitoyenne. Dans ces quelques pages, nous aborderons les expériences significatives vécues durant l'enfance à travers lesquelles se forge notre identité et se complexifie notre rapport au monde. Nous nous pencherons également sur l'impact de ces expériences sur le processus de développement de la passion envers la cause environnementale. Quelques pistes de réflexion seront également offertes aux éducatrices et éducateurs qui souhaitent contribuer au développement d'une identité écologique et favoriser l'émergence de la passion chez autrui. Dans un premier temps, il importe toutefois de définir ce qu'est la passion et de souligner son important rôle motivationnel dans l'engagement actif envers l'environnement. 


\section{La passion}

\section{Objet d'étude}

2 On retrouve dans la littérature philosophique deux visions contrastées de la passion (Rony, 1990). Pour certains comme Platon ou Spinoza, la passion est un mal allant à l'encontre de la raison. Passif, on y succombe aveuglément. Pour d'autres comme Rousseau ou Hegel, la passion, loin d'être contraignante et incontrôlable, constitue une énergie nécessaire au développement de notre plein potentiel. Récemment, Vallerand et ses collaborateurs (2003) ont tenté de réconcilier ces deux perspectives opposées en élaborant le Modèle dualiste de la passion.

\section{Passion et identité : le Modèle dualiste de la passion}

Selon ce modèle, la passion serait une vive inclination envers une activité (ou une cause) qui nous définit, que l'on aime, que l'on valorise et dans laquelle on investit régulièrement temps et énergie. La passion est si importante que nous en venons à nous identifier à celle-ci. Elle fait partie de nous, de qui nous sommes. Ainsi, un passionné de guitare se décrirait comme un guitariste, une passionnée d'environnement comme une écocitoyenne.

Le Modèle dualiste postule l'existence de deux types de passion: la passion harmonieuse ( $\mathrm{PH}$ ) et la passion obsessive (PO). Les deux types se distinguent par la façon dont l'activité passionnante est intériorisée dans l'identité de la personne. Une intériorisation de type autonome résulterait en une PH. Dans cette perspective, l'individu choisit librement et volontairement de s'engager dans l'activité passionnante, sans pression ni contraintes. L'activité s'harmonise avec les autres sphères de la vie du passionné : elle y prend une place centrale, mais non excessive. L'individu reste en contrôle de sa passion et se sent libre de choisir de s'engager ou non dans son activité. À l'opposé, la PO résulterait d'une intériorisation incontrôlée de l'activité dans l'identité d'un individu. Ce type d'intériorisation fait en sorte que l'individu ressent un désir incontrôlable de pratiquer l'activité qu'il aime. Il ne peut tout simplement pas s'en empêcher. Cette intériorisation renvoie donc à une pression interne incitant la personne à s'engager dans l'activité. Occupant une place disproportionnée dans la vie du passionné, l'activité peut mener à des conflits entre les différents domaines de vie. Les deux types de passion se distinguent également par leurs conséquences affectives, cognitives et comportementales. Ainsi, la PH mène généralement à des conséquences plus adaptatives (comme des émotions positives, une satisfaction de vie) que la PO qui peut se marquer par des comportements agressifs ou de mauvaises relations interpersonnelles. Plusieurs études réalisées dans divers contextes soutiennent le Modèle dualiste de la passion, notamment dans le domaine des jeux de hasard et d'argent (Castelda, Mattson, MacKillop, Anderson et Donovick, 2007), de la danse (Rip, Fortin et Vallerand, 2006), du travail (Carbonneau, Vallerand, Fernet et Guay, 2008), du sport (Vallerand, Rousseau, Grouzet et Grenier, 2006) et des loisirs (Stenseng, 2008). 


\section{Être passionné : les bienfaits pour l'environnement}

Bien que très peu d'études portant sur la passion envers la cause environnementale aient été menées jusqu'à présent, les recherches existantes semblent démontrer que la passion est une force motivationnelle importante pour expliquer l'implication environnementale. Certaines études ont entre autres montré que la prévalence de la passion est très élevée chez les individus impliqués d'une manière ou d'une autre en environnement. Par exemple, dans une série de trois études portant sur l'engagement en matière d'environnement, plus de $90 \%$ des participants étaient au moins modérément passionnés pour la cause (Gousse-Lessard, Vallerand, Carbonneau et Lafrenière, 2013). Il est important de souligner que ces participants étaient tous fortement impliqués envers l'environnement puisqu'ils étaient membres, employés ou bénévoles dans des organismes ou regroupements environnementaux. Ce haut taux de passionnés met en lumière l'important rôle de la passion dans ce type d'implication, les « non- passionnés » ne semblant pas s'investir dans de tels organismes.

6 Nos recherches (Gousse-Lessard et Vallerand 2012, Étude 2; Gousse-Lessard et coll., 2013) montrent également que les deux types de passion jouent un rôle différentiel sur le type d'engagement. Ainsi, la PH semble être associée à un large éventail de comportements environnementaux (des plus simples aux plus complexes) incluant l'activisme de type modéré qui peut se traduire par l'élaboration de pétitions, l'organisation de journée de sensibilisation aux enjeux environnementaux dans le quartier, l'organisation d'un système de covoiturage au travail. La PO, quant à elle, semble ne pas être associée aux gestes écologiques quotidiens considérés comme étant plus faciles (recyclage, achats de produits biologiques/équitables), mais seulement aux comportements demandant un surplus d'efforts tels l'auto-formation et l'activisme. Ces recherches montrent également que contrairement à la PH qui est peut être associée aux comportements activistes modérés, la PO est associée à la fois aux comportements activistes modérés et radicaux (par exemple, s'en prendre physiquement à un représentant d'une usine, ne plus parler à un membre de sa propre famille s'il n'accepte pas d'agir de façon écologique, grimper en haut d'un pont pour faire parler de la cause). Il est important de noter que ce type de radicalisme est normatif, c'est-à-dire que ces comportements ne sont jugés ni bons ni mauvais, mais simplement hors- normes. La passion semble donc être garante d'un fort engagement en matière d'environnement, les deux types de passion engendrant différents profils d'implication.

7 L'une de nos recherches (Gousse-Lessard et Vallerand, 2012) a également démontré que la passion est un meilleur prédicteur de comportements environnementaux que la motivation. Être motivé c'est bien, mais être passionné, c'est mieux! Pourquoi ? Ces deux construits diffèrent sur un point important : l'identification à l'activité. En effet, contrairement à la passion, la motivation n'est pas intériorisée dans l'identité de l'individu. Ainsi une personne peut être motivée à jouer au soccer avec ses amis seulement parce qu'elle y prend plaisir, sans que cette activité ne fasse partie intégrante de son identité. Dans ce cas-ci, l'activité n'est pas suffisamment importante pour que la personne se définisse par celle-ci. À l'opposé, pour un passionné, l'activité (ou la cause) importe tellement qu'elle est intégrée dans l'image qu'il entretient de luimême. Cette différence pourrait expliquer la supériorité du pouvoir prédicteur de la passion dans l'adoption d'une conduite écologique diversifiée. 
8 Ainsi, être passionné pour la cause environnementale semble être garant d'un fort engagement en matière d'environnement, soit par l'implication dans un organisme ou regroupement environnemental, par l'adoption d'une conduite écocitoyenne ou par l'activisme individuel et collectif. De quelle manière, alors, les éducatrices et éducateurs peuvent-ils transmettre leur passion?

\section{Comment favoriser le développement de la passion ?}

\section{Une étude sur les expériences de vie significatives}

Nous avons récemment mené une étude dont le but était d'étudier l'influence d'une expérience significative liée à l'environnement sur l'identification avec la nature et le développement de la passion pour la cause environnementale. Soixante-douze participants provenant du domaine de l'environnement (c'est-à-dire les membres, bénévoles ou employés dans des organismes environnementaux et individus provenant du milieu académique) nous ont raconté par écrit un souvenir d'enfance. Ce souvenir, positif ou négatif, devait concerner un événement lié à l'environnement (compris au sens large du terme) et devait être considéré par les participants comme hautement significatif et représentatif d'eux-mêmes. Ils devaient par la suite répondre à un court questionnaire concernant les émotions vécues pendant cet événement, le degré auquel ils sentaient que leurs besoins fondamentaux (comme l'autonomie, la compétence et l'affiliation: voir Deci et Ryan, 2002) étaient satisfaits lors de cet événement, leur connexion avec la nature (Nisbet, Zelenski et Murphy, 2009) une fois adulte, leur passion pour la cause environnementale (Vallerand et coll. 2003) et les conséquences notamment quant à leur conduite écologique et leur bien-être général (Diener, Emmons, Larsen et Griffin, 1985 ; Steger, Frazier, Oishi et Kaler, 2006).

La figure 1 résume les résultats de cette étude. En général, plus le souvenir était perçu comme positif (valence), plus les participants ont rapporté avoir vécu des émotions positives. En retour, plus ils ont ressenti que leurs besoins fondamentaux étaient satisfaits lors de l'événement. Par exemple, pendant l'événement, ils se sentaient libres d'agir et de penser comme ils le voulaient (besoin d'autonomie), ils se sentaient compétents (besoin de compétence) et ils se sentaient liés à une ou plusieurs personnes (besoin d'affiliation). Des années plus tard, cette satisfaction des besoins issue d'une expérience significative positive durant l'enfance semble avoir contribué positivement à une connexion profonde avec la nature. Cette connexion ou identification à la nature a été mesurée à l'aide d'énoncés tels «Ma relation avec la nature est une partie importante de qui je suis » et " Je me sens très connecté(e) à tous les êtres vivants et à la Terre ». Cette connexion à la nature est par la suite associée aux deux types de passion envers la cause environnementale, qui eux, sont reliés de façon différentielle aux conséquences. 
Figure 1 : Rôle des expériences significatives sur le développement de la passion envers la cause environnementale

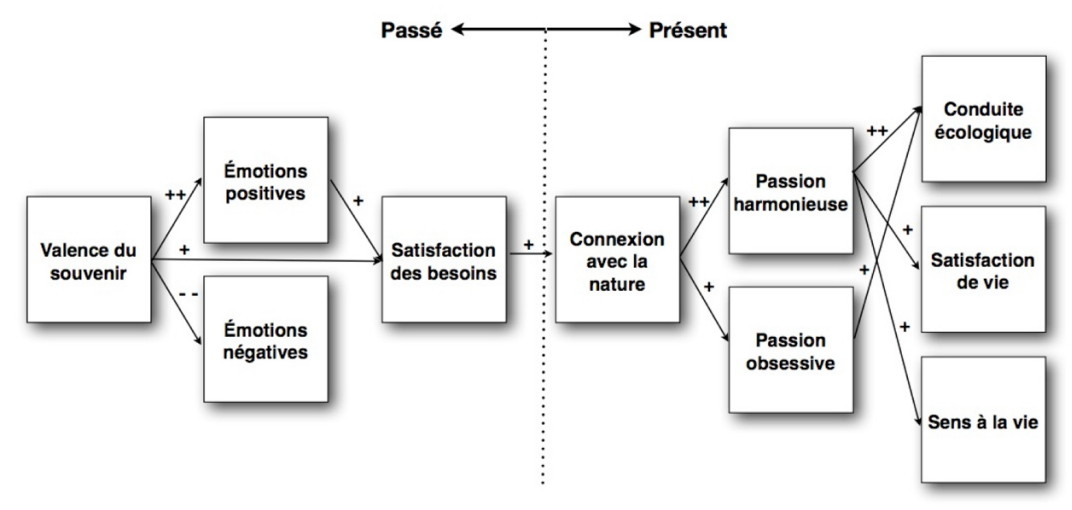

Figure 1 : Rôle des expériences significatives sur le développement de la passion envers la cause environnementale majorité $(90 \%)$ des participants nous ont spontanément raconté une expérience positive telle qu'une excursion en forêt à l'automne, la découverte d'animaux sur le bord du fleuve, la cueillette de fruits dans le verger, etc. plutôt que négative (catastrophe naturelle, perte d'une aire de jeu, détérioration d'un milieu). Les expériences positives en lien avec l'environnement resteraient peut-être plus accessibles en mémoire une fois adulte et contribueraient peut-être davantage à l'implication environnementale que les expériences négatives. De futures études devront examiner plus en profondeur le rôle différentiel de ces deux types d'expériences dans la construction de l'identité écologique et le développement de la passion envers la cause environnementale.

Il est aussi intéressant de noter que les deux types de passion sont associés de façon distincte aux conséquences. En effet, bien que les deux passions semblent mener à un certain engagement environnemental comme le recyclage, le compostage, l'utilisation de transport en commun, la sensibilisation des proches, l'implication dans un organisme environnemental ou encore l'achat de produits biologiques/équitables, seule la PH est reliée à la satisfaction de vie ainsi qu'à la perception que la vie a un sens. Ces résultats concordent avec les recherches antérieures menées dans divers domaines quant aux bienfaits de la PH. Alors que plusieurs affirment s'engager dans une cause afin de donner un sens à leur vie, il est intriguant de constater que les individus ayant une PO, malgré leur fort engagement, ne semblent pas trouver dans leur implication un tel sens. Être passionné pour une cause pourrait donc ne pas suffire: sans doute vaudrait-il mieux être passionné de façon harmonieuse pour en retirer tous les avantages. Il est important de souligner que la présente étude ne permet pas d'identifier les processus spécifiques menant aux deux types de passion. Puisqu'il semble que la PH soit davantage bénéfique (surtout sur le plan personnel), d'autres études devront assurément se pencher sur la question afin de déterminer quels processus favorisent l'émergence d'une telle passion.

13 Les résultats mettent aussi en lumière le rôle des émotions positives et de la satisfaction des besoins fondamentaux durant l'enfance dans la création d'une connexion profonde avec l'environnement. Que ce soit le souvenir d'une cabane dans un arbre, d'une journée de pêche avec papa ou des soirs de camping à la belle étoile, ces 
moments précieux où l'on se sent comblé et heureux semblent être un important creuset de construction de l'identité.

\section{Perspective éducative}

Bien que préliminaires, les résultats de l'étude nous offrent quelques pistes de réflexion quant au rôle de l'éducation relative à l'environnement dans la construction d'une identité écologique et le développement de la passion envers la cause environnementale. Dans une perspective éducative, il semblerait souhaitable de créer ces moments spéciaux de contact direct avec l'environnement afin de faire émerger une telle passion. Il est cependant difficile de prévoir si une activité ou une formation sera un jour perçue comme étant très significative pour l'enfant devenu adulte. Alors, en tant qu'éducatrices et éducateur, comment faciliter le bourgeonnement d'une passion pour l'environnement?

1. L'éducatrice ou l'éducateur pourrait intégrer à sa planification des expériences positives (c'est-à-dire non reliées à la détérioration d'un milieu) associées à un plus grand bien-être affectif, favorisant ainsi la connexion et l'identification à la nature et, du même souffle, le développement d'une passion.

2. L'éducatrice ou l'éducateur pourrait tenter de susciter des émotions positives (plaisir, émerveillement, sérénité, admiration) plutôt que négatives (peur, colère, tristesse, insatisfaction, impuissance). Par exemple, une approche uniquement basée sur les problèmes environnementaux, de par les émotions négatives pouvant y être attachées, serait pour le moins incomplète, voire contreproductive. L'exploration des possibles solutions aux problèmes, par exemple, devrait accompagner ce type d'approche afin d'engendrer le plus d'affects positifs possible.

3. L'éducatrice ou l'éducateur pourrait également prendre en compte la satisfaction des besoins fondamentaux dans l'élaboration d'activités formatrices. Par exemple, susciter une implication volontaire de l'individu en lui offrant des choix (ne pas être contrôlant) facilite la satisfaction du besoin d'autonomie. Les activités collaboratives en groupes facilitent la satisfaction du besoin d'affiliation. Les activités à la hauteur des moyens et habiletés des individus, à l'échelle locale (sans tenter de sauver le monde entier), facilitent la satisfaction du besoin de compétence. Des activités incluant ces composantes seraient donc bénéfiques pour le processus de développement de la passion envers l'environnement.

15 Bien sûr, il serait présomptueux de faire des recommandations basées sur une seule étude. Nous offrons ces quelques suggestions dans une perspective réflexive et dans la mesure où un certain nombre d'études antérieures réalisées dans divers domaines autres que l'environnement soutiennent ces propos. Nous croyons avant tout qu'il est important d'amorcer une réflexion quant au rôle des expériences de vie significatives vécues durant l'enfance sur le développement de la passion pour la cause environnementale, ainsi que le lien étroit existant entre l'identité écologique, la passion, et l'engagement en matière d'environnement. Ces expériences, lorsque positives, semblent fortifier et dynamiser notre rapport au monde et favoriser l'intériorisation de l'environnement dans l'image que nous entretenons de nous-même : l'environnement c'est nous, il faut en prendre soin. 


\section{Conclusion}

La passion semble être une force motivationnelle particulièrement importante dans l'explication d'un engagement intense en matière d'environnement. Dans ce texte, nous avons souligné quelques mécanismes possibles pouvant expliquer les liens complexes entre les expériences de vie significatives, l'identité écologique et une conduite écocitoyenne. Ce domaine de recherche est toutefois jeune et plusieurs questions non répondues pourront alimenter les recherches futures. Il serait important par exemple de mieux cerner les processus d'intériorisation de la passion dans l'identité. Nous ne comprenons pas encore clairement ce qui amène les gens à développer une passion harmonieuse plutôt qu'obsessive, ni de quelle manière les expériences de vie négatives (c'est-à-dire reliées à une perte ou à la dégradation de l'environnement) modulent notre rapport avec la nature et du même coup notre engagement environnemental. Le rôle de la satisfaction des besoins fondamentaux dans les deux types de passion pour l'environnement demeure une autre question à approfondir. Il serait important par exemple de savoir dans quelle mesure les sentiments d'autonomie, de compétence et d'affiliation vécus dans le contexte d'une expérience de vie significative ont une influence sur le développement initial et continu d'une passion pour la cause environnementale. Les réponses à ces questions et d'autres encore aideront sans doute à démystifier les interrelations complexes entre l'environnement et la transformation de notre identité. Dans un monde où l'engagement environnemental semble devenu une nécessité, l'éducation relative à l'environnement pourrait être un outil merveilleux afin de faciliter le développement de la passion en matière d'environnement.

\section{BIBLIOGRAPHIE}

Carbonneau, N., Vallerand, R. J., Fernet, C. et Guay, F. (2008). The role of passion for teaching in intra and interpersonal outcomes. Journal of Educational Psychology, 100, 977-987.

Castelda, B.A., Mattson, R.E., MacKillop, J., Anderson, E.J. et Donovick, P.J. (2007).

Psychometric validation of the Gambling Passion Scale (GPS) in an English-speaking university sample. International Gambling Studies, 7, 173-182.

Deci, E. L., Ryan, R. M. (Eds.). (2002). Handbook of self-determination research. Rochester, NY : University of Rochester Press.

Diener, E., Emmons, R. A., Larsen, R. J. et Griffin, S. (1985). The Satisfaction With Life Scale. Journal of Personality Assessment, 49, 71-76.

Gousse-Lessard, A-S., Vallerand, R.J. (2012, janvier). When passion is greener than motivation. Communication affichée présentée au 13e congrès international de la Society for Personality and Social Psychology (SPSP), San Diego, États-Unis. 
Gousse-Lessard, A-S., Vallerand, R.J., Carbonneau, N. et Lafrenière, M-A. K. (2013). The role of passion in mainstream and radical behaviors : A look at environmental activism. Journal of Environmental Psychology, 35, 18-29.

Nisbet, E. K. L., Zelenski, J. M., Murphy, S. A. (2009). The nature relatedness scale : LLinking individuals' connection with nature to environmental concern and behaviour. Environment and Behavior, 41, 715- 740.

Rip, B., Fortin, S., Vallerand, R. J. (2006). The relationship between passion and injury in dance students. Journal of Dance Medicine \& Science, 10, 14-20.

Rony, J.-A. (1990). Les passions [The passions]. Paris : Presses Universitaires de France.

Steger, M. F., Frazier, P., Oishi, S., Kaler, M. (2006). The meaning in life questionnaire : Assessing the presence of and search for meaning in life. Journal of Counseling Psychology, Vol 53, 80-93.

Stenseng, F. (2008). The two faces of leisure activity engagement : Harmonious and obsessive passion in relation to intrapersonal conflict and life domain outcomes. Leisure Sciences, 30, 465-481.

Suzuki, D. (2002). Message from the chair. In David Suzuki Foundation (Ed.), Annual report 2001 (p.3). Repéré à www.davidsuzuki.org/publications/downloads/2001/DSF \%3DARfinal11.pdf

Vallerand, R.J., Blanchard, C., Mageau, G.A., Koestner, R., Ratelle, C.F., Léonard, M., Gagné, M. et Marsolais, J. (2003). Les passions de l'âme : On obsessive and harmonious passion. Journal of Personality and Social Psychology, 85, 756-767

Vallerand, R. J., Rousseau, F. L., Grouzet, F. M. E., Dumais, A., \& Grenier, S. (2006). Passion in sport : A look at determinants and affective experiences. Journal of Sport and Exercise Psychology, 28, 454-478.

\section{INDEX}

Mots-clés : environnement, engagement environnemental, passion, expérience de vie, identité Keywords : environment, environmental commitment, passion, life experience, identity

\section{AUTEURS}

\section{ANNE-SOPHIE GOUSSE-LESSARD}

Anne-Sophie Gousse-Lessard a complété le programme court de deuxième cycle en éducation relative à l'environnement à l'Université du Québec à Montréal. Elle poursuit depuis 2009 ses études au doctorat en psychologie sociale/environnementale au sein du Laboratoire de recherche sur le comportement social. Ses recherches portent principalement sur la passion pour la cause environnementale et l'activisme. Son implication l'amène également à écrire des billets de blogues pour Écologistik, une entreprise spécialisée en gestion d'événements écoresponsables.

\section{DANIEL LALANDE}

Professeur à l'Université du Québec à Chicoutimi dans le département des sciences de la santé. Ses recherches portent sur la satisfaction des besoins psychologiques, les déterminants de la passion, le gambling et l'autorégulation du comportement. 
ROBERT J. VALLERAND

Professeur au département de psychologie et directeur du Laboratoire de Recherche sur le Comportement Social à l'Université du Québec à Montréal. Son expertise porte notamment sur la motivation sociale et la passion pour une activité. Auteur de plusieurs livres et publications dans ce domaine, il a aussi siégé en tant que président pour la Société québécoise de recherche en psychologie, la Société canadienne de psychologie et l'Association internationale de psychologie positive. 